\title{
Daucosterol pretreatment ameliorates myocardial ischemia reperfusion injury via ROS-mediated NLRP3 inflammasome activation
}

\author{
Guixiang Zhao', Xiaoyun $\mathrm{Ma}^{2}$, Juledezi·Hailati², Zhen Bao², Maerjiaen·Bakeyi², \\ Zhiqiang Liü ${ }^{2 *}$ \\ ${ }^{1}$ Department of Cardiology, The Sixth Affiliated Hospital of Xinjiang Medical University, ${ }^{2}$ Department of Cardiology, The First \\ Affiliated Hospital of Xinjiang Medical University, Urumqi, Xinjiang Uygur Autonomous Region 830054, China
}

*For correspondence: Email: ZhiqiangLiudjk@163.com; Tel: +86-991-4366851

Sent for review: 10 March 2020

Revised accepted: 29 April 2020

\begin{abstract}
Purpose: To determine the involvement of NLRP3 signaling pathway in the preventive role of daucosterol in acute myocardial infarction (AMI).

Methods: H9C2 cells were pretreated with daucosterol before hypoxia/reoxygenation (HR) injury. Myocardial ischemia reperfusion (IR) was established in male $S D$ rats, followed by reperfusion. Myocardial infarct size was measured. The serum levels of creatine kinase (CK), lactate dehydrogenase $(L D H)$, total superoxide dismutase (T-SOD), and malondialdehyde (MDA) were determined using commercial kits. NLRP3 inflammasome activation was assessed by western blotting.

Results: Myocardial infarct size was smaller after IR injury in rats pretreated with daucosterol (10 and $50 \mathrm{mg} / \mathrm{kg}$ ) than that pretreated with daucosterol ( 0 and $1 \mathrm{mg} / \mathrm{kg}$ ). The increase in $\mathrm{LDH}$, CK, and MDA levels after IR injury was reduced following daucosterol pretreatment. Reactive oxygen species (ROS) production increased, whereas T-SOD activity decreased after IR injury. These changes were prevented by pretreatment of daucosterol $(10$ and $50 \mathrm{mg} / \mathrm{kg})$. Protein expression of NLRP3 inflammasome increased after IR injury in $\mathrm{H} 9 \mathrm{C} 2$ cells while pretreatment with daucosterol inhibited the upregulation of NLRP3 inflammasome.

Conclusion: The cardioprotective effect of daucosterol pretreatment appears to be mediated via the inactivation of ROS-related NLRP3 inflammasome, suggesting that daucosteol might be a potential therapeutic drug for AMI.
\end{abstract}

Keywords: Daucosterol, Myocardial ischemia, Reperfusion injury, Reactive oxygen species, NLRP3 inflammasome

\begin{abstract}
This is an Open Access article that uses a fund-ing model which does not charge readers or their institutions for access and distributed under the terms of the Creative Commons Attribution License (http://creativecommons.org/licenses/by/4.0) and the Budapest Open Access Initiative (http://www.budapestopenaccessinitiative.org/read), which permit unrestricted use, distribution, and reproduction in any medium, provided the original work is properly credited.
\end{abstract}

Tropical Journal of Pharmaceutical Research is indexed by Science Citation Index (SciSearch), Scopus, International Pharmaceutical Abstract, Chemical Abstracts, Embase, Index Copernicus, EBSCO, African Index Medicus, JournalSeek, Journal Citation Reports/Science Edition, Directory of Open Access Journals (DOAJ), African Journal Online, Bioline International, Open-J-Gate and Pharmacy Abstracts

\section{INTRODUCTION}

Acute myocardial infarction (AMI) is one of the most serious complications of cardiovascular disease [1]. Due to the interventional therapy of percutaneous coronary, bypass grafting of coronary artery, and long-term treatment with inhibitors of angiotensin-converting enzyme, clotbusting agents, and $\beta$-blockers, increasing patients have survived AMI [2]. However, 
patients eventually develop heart failure due to myocardial dysfunction and left ventricular remodeling [3]. Ischemia reperfusion (IR) injury results from the suspension of blood supply that subsequently restored to an organ [4]. Reduced oxygen supply during ischemia can cause cardiac dysfunction and cardiomyocyte death, which elevated the release of ROS during reperfusion $[5,6]$.

Increased ROS levels lead to tissue dysfunction by inducing peroxidation of lipids and proteins, mitochondria dysfunction, as well as DNA damage [7]. During IR, ROS production activates tissue inflammation and promotes immunoreaction via activating NLRP3 inflammasome [8]. Following activation, NLRP3 interacts with the adaptor protein ASC to recruit procaspase-1 monomers, which thus leads to the activation of caspase-1[9]. Recent research has revealed that the GSDMD, the substrate of active caspase- 1 is enzymatically cleaved by active caspase-1 into two fragments (the $\mathrm{N}$ and $\mathrm{C}$ domains) [10]. Upregulation of GSDMD-N induces pyroptosis and promote cardiomyocyte death during IR injury [11].

Daucosterol is a natural glucoside of $\beta$-sitosterol and synthesized from plants [12]. Recent studies have revealed that daucosterol has effects on various biological process, and has anti-cancer, anti- inflammatory, and neuroprotective effects $[3,12]$. In mice, daucosterol prevented clinical symptoms of colitis and inhibited ROS production, macrophage infiltration, and the expression of pro-inflammatory cytokines caused by dextran sulfate sodium (DSS), indicating its anti-inflammatory effect [12]. However, there is no evidence regarding the effect of daucosterol on AMI.

Therefore, this study aimed to reveal the biological activity of daucosterol following myocardial IR injury, and the underlying signaling pathway.

\section{EXPERIMENTAL}

\section{Animals}

Male Sprague-Dawley (SD) rats, weighing $275 \pm$ $25 \mathrm{~g}, 7 \pm 1$ weeks old, were obtained from Hunan Shrek Jingda Experimental Animal Co. Ltd (Hunan Province, China). Before the experiment, all rats were given normal laboratory rat feed and water and maintained in a controlled environment (24 $\pm 1{ }^{\circ} \mathrm{C}, 55 \pm 15 \%$ humidity, 12-h light/12-h dark cycle), for 1 week to adapt to the environment. All experiments procedures were carried out in compliance with ethical standards under a protocol approved by the institutional Ethics Committee (approval no. 2018035), and executed according to the Guide for the Care and Use of Laboratory Animals published by United States NIH (no. 85-23, 1996).

\section{Animal model of AMI and groups}

Myocardial IR was achieved by encircling the left anterior descending coronary artery (LAD; 30 $\mathrm{min})$, followed by reperfusion $(1 \mathrm{~h})$. The $S D$ rats were anesthetized by urethane $(1 \mathrm{~g} / \mathrm{kg}$, Qingxi, Shanghai, China) and the chest was opened. Ischemia was induced by placing a slipknot with silk suture at the distal one-third of the LAD (30 min). Subsequently, the myocardium was reperfused for $1 \mathrm{~h}$ by releasing the slipknot. The same procedures were conducted without tying the suture at the LAD, for sham group.

The rats were grouped ( $\mathrm{n}=10$ per group): I: Sham rats; II: AMI rats; III: AMI rats were pretreated with daucosterol $(1 \mathrm{mg} / \mathrm{kg})$; IV: AMI rats were pretreated with daucosterol at a dose of $10 \mathrm{mg} / \mathrm{kg}$; V, AMI rats were pretreated with daucosterol (50 mg/kg). Daucosterol was administered through the intragastric route, three times per week for 2 weeks before operation. At the end of the experiments, the rats were euthanized by pentobarbital sodium $(100 \mathrm{mg} / \mathrm{kg}$ ) and then blood sample and heart tissues were collected.

\section{Blood and heart tissue collection}

A volume of $500 \mu \mathrm{L}$ blood samples from the tail vein of rats after reperfusion was centrifuged at $1000 \mathrm{rpm}$. The supernatants were collected in 1$\mathrm{ml}$ tubes and stored at $-80{ }^{\circ} \mathrm{C}$. The heart was isolated after reperfusion for $1 \mathrm{~h}$. A portion of each heart specimen was fixed and kept at $4{ }^{\circ} \mathrm{C}$ for ultrastructure detection. Another portion of each specimen was embedded in paraffin wax after fixing in $4 \%$ paraformaldehyde. The remaining part of heart was deep-frozen by liquid nitrogen and preserved at $-80^{\circ} \mathrm{C}$.

\section{Measurement of myocardial infarct size}

Triphenyl tetrazolium chloride (TTC) staining was used to measure myocardial infarct size. The heart tissues were cut into slices (5-mm) and incubated in TTC (1\%), and photographed using a digital camera (Canon, Japan). Following TTC staining, the red portion of the heart represent ischemic but not infarct tissue, while the white portion represents infarcted myocardium. Image Pro Plus software was used for the measurement of the size of myocardial infarct areas. 
Cell culture and hypoxia/reoxygenation (H/R) injury model

Rat cardiomyocyte cell lineH9C2 was purchased from ATCC (Manassas, VA, USA.). High-glucose DMEM were supplemented with $10 \%(\mathrm{v} / \mathrm{v})$ heatinactivated FBS and antibiotics (Gibco/Invitrogen). The cells were incubated in a humidified incubator $\left(5 \% \mathrm{CO}_{2} ; 37^{\circ} \mathrm{C}\right)$.

H9C2 cells were cultured in sterile 6-well plates $\left(1 \times 10^{7}\right.$ cells/well), and added with daucosterol that dissolved in dimethyl sulfoxide for $24 \mathrm{~h}$; this was the $H / R+D A$ group. The control and $H / R$ groups were cultured in normal medium for $24 \mathrm{~h}$.

The medium was then changed to Krebs-Ringer bicarbonate buffer and the $H / R$ cells were incubated in an anoxic environment $\left(95 \% \mathrm{~N}_{2}\right.$ and $5 \% \mathrm{CO}_{2}$ for $6 \mathrm{~h}$, whereas the control cells was cultured under normal condition for 6 hours. Following this, all groups were cultured in normal medium and maintained at $5 \% \mathrm{CO}_{2}$ and $95 \%$ air for $5 \mathrm{~h}$ to effect reoxygenation injury.

Assay of CK, LDH, T-SOD, and MDA in serum

As described above, blood serum was collected and serum CK (ab155901, Abcam) and leakage of myocardial lactate LDH were determined at 450 $\mathrm{nm}$ (ab102526, Abcam) according to manufacturer's instructions. Quantification of TSOD activity at $450 \mathrm{~nm}$ (ab65354, Abcam) and MDA at $532 \mathrm{~nm}$ (ab118970, Abcam) were carried out using commercial assay kits.

\section{Fluorescence microscopy}

Cells were seeded in 6-well plates. After treatment, cells were fixed and then washed with PBS three times and stained by Hoechst 33258 solution (Beyotime), which was finally observed using fluorescence microscopy (Olympus IX81).

\section{Flow cytometry}

Apoptosis of $\mathrm{H} 9 \mathrm{C} 2$ cell was determined using flow cytometry. $\mathrm{H} 9 \mathrm{C} 2$ cells were collected and centrifuged. After washing with PBS, the cells were analyzed using Annexin V-FITC apoptosis detection kit [13]and analyzed using flow cytometry (Becton Dickinson, Mountain View, CA, USA).

\section{Western blotting analysis}

Total proteins were extracted from myocardial tissue and $\mathrm{H} 9 \mathrm{C} 2$ cells and a BCA protein assay kit (Pierce) was used to measure concentration. Fifty micrograms of total protein were electrophoresed on a $10 \%$ SDS-PAGE and then transferred to membranes (Millipore Co., Bedford, USA), which were blocked and incubated overnight at $4{ }^{\circ} \mathrm{C}$ with primary antibodies: Blc2 (CST3498, 1:1500 dilution), caspase 3 (CST9665, 1:1500 dilution), cleaved caspase 3 (CST9664, 1:1000 dilution), NLRP3 (CST15101, 1:800 dilution), activated caspase-1 (p20) (SC12128, 1:500 dilution), caspase-1 (CST2225, 1:1000 dilution), GSDMD-N (AF4012, 1:600 dilution), and $\beta$-actin (CST3700, 1:5000 dilution).

Thereafter, the membranes were incubated with secondary antibody, and bands were detected and quantified.

\section{Statistical analysis}

Statistical analysis was performed using GraphPad Prism 8.1.0 software. Data were shown as mean \pm standard error of mean. Comparison analysis involving more than two groups were using one-factor analysis of variance; differences between individual groups were analyzed using Scheffe's test. A p-value < 0.05 was considered statistically significant.

\section{RESULTS}

\section{Daucosterol reduced myocardial infarction in rats after IR injury}

Infarcts in rat hearts were stained using TTC and measured. No infarction was found in the sham group, whereas infarct areas were displayed in the AMI group. The infarct size was smaller in 10 $\mathrm{mg} / \mathrm{kg}$ and $50 \mathrm{mg} / \mathrm{kg}$ daucosterol-treated groups than that in the AMl group, whereas $1 \mathrm{mg} / \mathrm{kg}$ of daucosterol did not reduce infarct size as compared with the AMI group (Figure $1 \mathrm{~A}$ ).

The elevation of LDH and CK levels were found in rats with IR, but this elevation was inhibited in rats pretreated with $10 \mathrm{mg} / \mathrm{kg}$ and $50 \mathrm{mg} / \mathrm{kg}$ daucosterol (Figure $1 \mathrm{~B}$ and $\mathrm{C}$ ).

\section{Daucosterol reduced ROS production in rats after IR injury}

Serum MDA levels were increased in rats following IR injury. However, pretreatment with daucosterol(10 and $50 \mathrm{mg} / \mathrm{kg}$ ) inhibited the elevation of MDA levels, whereas $1 \mathrm{mg} / \mathrm{kg}$ of daucosterol had no obvious effect on serum MDA levels (Figure 2 A).. T-SOD activity was reduced in $\mathrm{AMI}$ rats, but restored in rats pretreated with 10 and $50 \mathrm{mg} / \mathrm{kg}$ daucosterol (Figure 2 B).

Trop J Pharm Res, May 2020; 19(5): 1033 
A

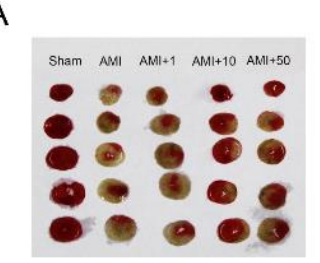

C

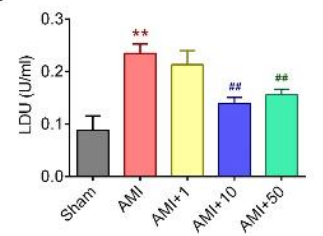

B

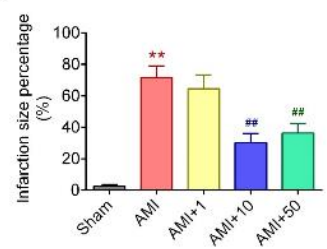

D

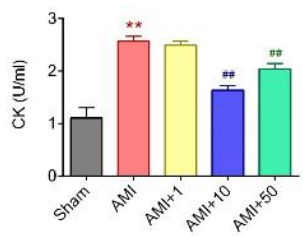

Figure 1: Daucosterol attenuated myocardial infarction after IR injury. (A) The myocardial infarct was stained using TCC; (B) the size calculated as a percentage of the area; serum $\mathrm{LDH}(\mathrm{C})$ and $\mathrm{CK}(\mathrm{D})$ level was measured using a commercial kit; ${ }^{* *} p<0.01$ vs. sham; ${ }^{\prime \prime} p<0.01$ vs. AMI

A

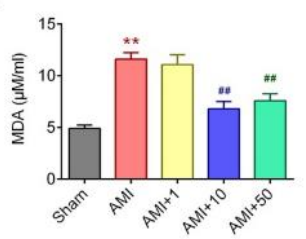

B

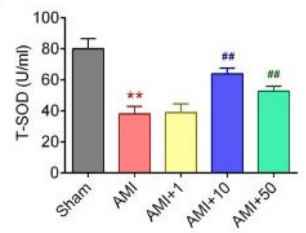

Figure 2: Daucosterol reduced oxidative stress after IR injury. (A) T-MDA (A) and SOD level (B) were measured using a commercial kit; ${ }^{* *} p<0.01$ vs. sham; $\#^{\#} p<0.01$ vs. AMI

\section{Daucosterol protected H/R injury by reducing ROS production and suppress apoptosis of H9C2 cells}

The fluorescence intensity of ROS was significantly enhanced in $\mathrm{H} / \mathrm{R}$-treated $\mathrm{H} 9 \mathrm{C} 2$ cells, while the addition of daucosterol decreased ROS fluorescence intensity (Figure $3 \mathrm{~A}$ ). The proportion of apoptotic cells was increased in H/R-treated H9C2 cells. In cells pretreated with daucosterol, the number of apoptotic cells induced by $\mathrm{H} / \mathrm{R}$ was significantly reduced (Figure $3 \mathrm{~B})$.

The expression of $\mathrm{Bcl} 2$ was downregulated after $H / R$, and daucosterol inhibited $H / R$-induced downregulation of $\mathrm{Bcl} 2$ expression. Caspase-3 expression showed no significant change by $H / R$ or daucosterol pretreatment, but cleaved caspase-3 expression was increased following $H / R$, which was inhibited by pretreatment of daucosterol (Figure $3 \mathrm{C}$ ).

A
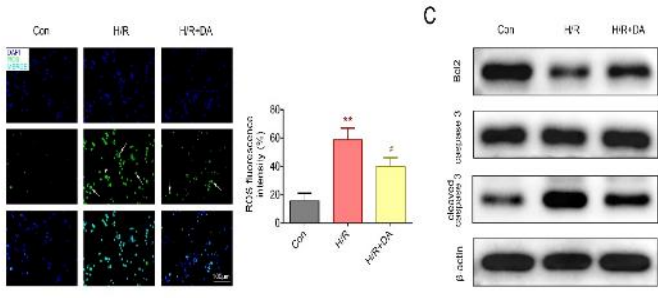

B
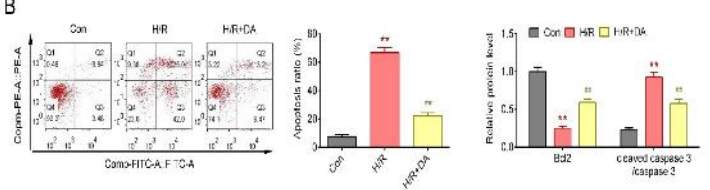

Figure 3: Daucosterol reduced ROS production and suppressed cell apoptosis after H/R injury. (A) Cells were stained by Hoechst 33258 to detect ROS production (200x); (B) Measurement of cell apoptosis was achieved by flow cytometry; (C) Protein expression was determined by western blotting; ${ }^{* *} p<0.01$ vs. control; ${ }^{\#} p<0.05$ vs. H/R; ${ }^{\#} p<0.01$ vs. H/R

\section{Daucosterol inhibited NLRP3 inflammasome activation after IR injury}

NLRP3 inflammasome activation (NLRP3, caspase- 1 , activated caspase- 1 , and GSDMD-N) was measured using western blotting, both in $\mathrm{H} 9 \mathrm{C} 2$ cells and in AMI rats. In H/R cells, the expression of NLRP3 inflammasome was significantly upregulated after $H / R$, and these upregulations were inhibited in cells pretreated with daucosterol. (Figure 4A). In rat heart tissue, upregulations of NLRP3 inflammasome were observed in rats following IR injury. Pretreatment with 10 and $50 \mathrm{mg} / \mathrm{kg}$ daucosterol prevented the increases in expression of NLRP3 inflammasome. However, no obvious biological effects were observed in rats treated with 1 $\mathrm{mg} / \mathrm{kg}$ daucosterol (Figure 4B).

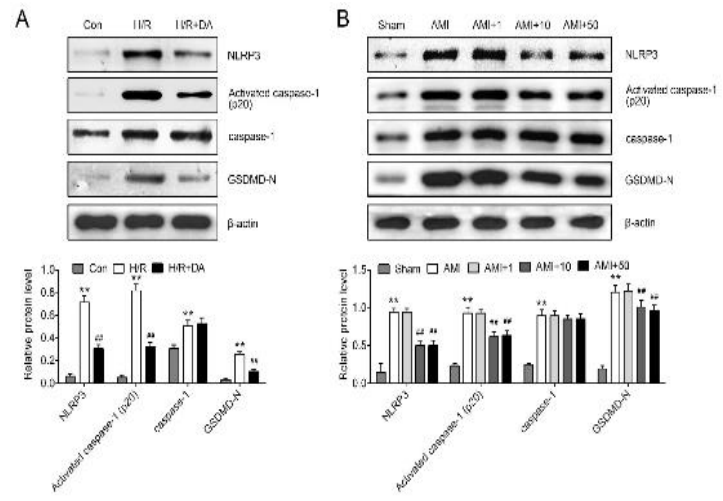

Figure 4: Daucosterol inhibited NLRP3 inflammasome activation after $H / R$ or IR injury. (A) The protein expressions in $\mathrm{H} 9 \mathrm{C} 2$ cells $(\mathrm{A})$ and $\mathrm{AMI}$ rats (b) were detected by western blotting; ${ }^{* *} p<0.01 \mathrm{vs}$. control/Sham; ${ }^{\# \#} p<0.01$ vs. H/R or AMI 


\section{DISCUSSION}

It is known that myocardial IR injury increases the morbidity and mortality of AMI patients, and that reperfusion is the standard therapy for AMI [14]. Recently, more and more studies have focused on identifying effective cardioprotective treatments to prevent IR injury $[15,16]$. This the first evidence investigating the cardioprotective effect of daucosterol on IR injury. The results demonstrated that pretreatment with daucosterol reduced myocardial infarct size in rats following IR injury, and that this effect is likely mediated via ROS-related NLRP3 inflammasome activation.

Ischemia and reperfusion elicit cardiomyocyte injury [17]. Fewer apoptotic cells were observed in $\mathrm{H} 9 \mathrm{C} 2$ cells pretreated with daucosterol than in $\mathrm{H} / \mathrm{R}$ cardiomyocytes. Moreover, LDH and CK are effective biomarkers of myocardial injury [18]. In rats pre-treated with daucosterol (10 and 50 $\mathrm{mg} / \mathrm{kg}$ ), serum LDH and CK levels, as well as infarct size, were lower, whereas no difference was observed in rats treated with $1 \mathrm{mg} / \mathrm{kg}$ of daucosterol when compared to AMI rats. These in vitro and in vivo data indicate that medium and high doses of daucosterol might prevent myocardial injury from IR. The signaling pathway underlying the cardioprotective effects of daucosterol was investigated. IR injury is mediated by several factors, and elevated ROS production occurs particularly at reperfusion [5]. ROS production was increased after $\mathrm{H} / \mathrm{R}$ injury in $\mathrm{H} 9 \mathrm{C} 2$ cells, which has also been demonstrated in previous findings [19]. Reduction in ROS fluorescence intensity was observed in cells pretreated with daucosterol, demonstrating that daucosterol inhibited $\mathrm{H} / \mathrm{R}$-induced ROS generation. The activity of T-SOD was reduced and serum MDA levels was enhanced after IR injury, indicating an increase in oxidative stress [20,21]. Pretreatment of cells with daucosterol reduced ROS generation and serum MDA levels, and enhanced T-SOD activity. These findings showed that daucosterol prevented cardiomyocyte injury by reducing oxidative stress.

It is well accepted that elevation of ROS production activates the NLRP3 inflammasome [22]. Previous studies have reported that ROSmediated NLPR3 inflammasome activation is involved in many different signaling pathways, such as MAPK, NF-kB, ERK1/2, and Nrf2 $[6,16,23,24]$. The data in this study revealed that NLRP3 expression was upregulated both in vitro and in vivo after IR or H/R injury. Furthermore, the upregulation of NLRP3 was inhibited by pretreatment with daucosterol, confirming that the cardioprotective effects of daucosterol were mediated by activation of the ROS-NLRP3 inflammasome signaling pathway.

Furthermore, protein expressions of activated caspase-1 and its substrate GSDMD-N, an NLPR3 inflammasome protein [10], were increased after IR or $H / R$ injury, indicating activation of pyroptosis [25]. Daucosterol inhibited the activation of caspase-1, resulting in the inactivation of pyroptosis. To some extent, these results were consistent with data showing smaller infarct size and fewer apoptotic cells in the daucosterol pre-treated group when compared with controls, both in vivo and in vitro.

\section{Limitations of the study}

The limitation of this study is that we were unable to pinpoint the underlying protective mechanism of daucosterol in ROS-NLRP3 activation. Further analysis is needed to identify the exact signaling pathway involved in the cardioprotective effect of daucosterol.

\section{CONCLUSION}

This is the first primary evidence showing that the protective effect of daucosterol on myocardial infarction is mediated via inactivation of NLRP3 inflammasome. This finding demonstrates that daucosterol may be a potential therapeutic agent for the prevention of IR injury. However, clinical studies are required to validate this statement.

\section{DECLARATIONS}

\section{Acknowledgement}

This work was supported by the National Science Foundation of China (Grant no. 81760062).

\section{Conflict of interest}

No conflict of interest is associated with this work.

\section{Contribution of authors}

We declare that this work was done by the authors named in this article and all liabilities pertaining to claims relating to the content of this article will be borne by the authors.

\section{Open Access}

This is an Open Access article that uses a funding model which does not charge readers or their institutions for access and distributed under the terms of the Creative Commons Attribution License (http://creativecommons.org/licenses/by/ 
4.0) and the Budapest Open Access Initiative (http://www.budapestopenaccessinitiative.org/rea d), which permit unrestricted use, distribution, and reproduction in any medium, provided the original work is properly credited.

\section{REFERENCES}

1. Members ATF, McMurray JJV, Adamopoulos S, Anker $S D$, Auricchio A, Böhm $M$, Dickstein $K$, Falk $V$, Filippatos G, Fonseca $C$ et al. ESC Guidelines for the diagnosis and treatment of acute and chronic heart failure. Eur J Heart Fail2012. 2012; 14(8): 803-869.

2. Sutton MG, Sharpe $N$. Left ventricular remodeling after myocardial infarction: pathophysiology and therapy. Circulation 2000; 101(25): 2981-2988.

3. Jiang L-h, Yuan X-I, Yang N-y, Ren L, Zhao F-m, Luo B$x$, Bian Y-y, Xu J-y, Lu D-x, Zheng Y-y et al. Daucosterol protects neurons against oxygen-glucose deprivation/reperfusion-mediated injury by activating IGF1 signaling pathway. J Steroid Biochem 2015; 152:45-52.

4. Hausenloy DJ, Yellon DM. Myocardial ischemiareperfusion injury: A neglected therapeutic target. J Clin Invest 2013; 123(1): 92-100.

5. Cadenas S. ROS and redox signaling in myocardial ischemia-reperfusion injury and cardioprotection. Free Radical Bio Med 2018; 117:76-89.

6. Zhang $D$, Hou L, Peng W. Tangeritin attenuates oxidative stress, apoptosis and inflammation in cadmium-induced cardiotoxicity in rats by activating Nrf2 signaling pathway. Trop J Pharm Res 2019; 17(12): 2421.

7. Sho $T, X U \mathrm{~J}$. Role and mechanism of ROS scavengers in alleviating NLRP3-mediated inflammation. Biotechnol Appl Bioc 2019; 66(1): 4-13.

8. Bryant C, Fitzgerald KA. Molecular mechanisms involved in inflammasome activation. Trends Cell Biol 2009; 19(9): 455-464.

9. Danielski LG, Giustina $A D$, Bonfante S, Barichello $T$, Petronilho F. The NLRP3 Inflammasome and lts Role in Sepsis Development. Inflammation 2019;1-8.

10. Shi J, Zhao $Y$, Wang $K$, Shi $X$, Wang $Y$, Huang $H$, Zhuang $Y$, Cai T, Wang F, Shao F. Cleavage of GSDMD by inflammatory caspases determines pyroptotic cell death. Nature 2015; 526(7575): 660-665.

11. Ye B, Chen X, Dai S, Han J, Liang X, Lin S, Cai X, Huang $Z$, Huang $W$. Emodin alleviates myocardial ischemia/reperfusion injury by inhibiting gasdermin $D$ mediated pyroptosis in cardiomyocytes. Drug Des Devel Ther 2019; 13:975-990.

12. Jang J, Kim S-M, Yee S-M, Kim E-M, Lee E-H, Choi H-R, Lee Y-S, Yang W-K, Kim H-Y, Kim K-H et al. Daucosterol suppresses dextran sulfate sodium (DSS)induced colitis in mice. Int Immunopharmacol 2019; 72:124-130.

13. Yan H-l, Xue G, Mei Q, Wang Y-z, Ding F-x, Liu M-F, Lu $M-H$, Tang $Y, Y u H-y$, Sun S-h. Repression of the miR-
17-92 cluster by $p 53$ has an important function in hypoxia-induced apoptosis. EMBO J 2009; 28(18): 2719-2732.

14. O'Gara PT, Kushner FG, Ascheim DD, Casey DE, Chung MK, de Lemos JA, Ettinger SM, Fang JC, Fesmire FM, Franklin BA et al. 2013 ACCF/AHA Guideline for the Management of ST-Elevation Myocardial Infarction: A Report of the American College of Cardiology Foundation/American Heart Association Task Force on Practice Guidelines. J Am Coll Cardiol 2013; 61(4): e78e140.

15. Yu D, Li M, Tian Y, Liu J, Shang J. Luteolin inhibits ROSactivated MAPK pathway in myocardial ischemia/reperfusion injury. Life Sci 2015; 122 : 15-25.

16. Jun JH, Shim J-K, Oh JE, Shin E-J, Shin E, Kwak Y-L. Protective Effect of Ethyl Pyruvate against Myocardial Ischemia Reperfusion Injury through Regulations of ROS-Related NLRP3 Inflammasome Activation. Oxid Med Cell Longev 2019; 2019: 4264580-4264580.

17. Vanden Hoek TL, Shao Z, Li C, Zak R, Schumacker PT, Becker LB. Reperfusion injury on cardiac myocytes after simulated ischemia. Am J Physiol 1996; 270 (4 Pt 2): H1334-H1341.

18. Adams JE, 3rd, Abendschein DR, Jaffe AS. Biochemical markers of myocardial injury. Is MB creatine kinase the choice for the 1990s? Circulation 1993; 88(2): 750-763.

19. Zhou T, Prather ER, Garrison DE, Zuo L. Interplay between ROS and Antioxidants during IschemiaReperfusion Injuries in Cardiac and Skeletal Muscle. Int J Mol Sci 2018; 19(2): 417.

20. Cherian DA, Peter $T$, Narayanan A, Madhavan SS, Achammada S, Vynat GP. Malondialdehyde as a Marker of Oxidative Stress in Periodontitis Patients. J Pharm Bioallied Sci 2019; 11(Suppl 2): S297-S300.

21. McCord JM, Edeas MA. SOD, oxidative stress and human pathologies: a brief history and a future vision. Biomed Pharmacother 2005; 59(4): 139-142.

22. Zheng Q, Ren Y, Reinach PS, She Y, Xiao B, Hua S, Qu $J$, Chen W. Reactive oxygen species activated NLRP3 inflammasomes prime environment-induced murine dry eye. Exp Eye Res 2014; 125:1-8.

23. Liu J, Mao C, Dong L, Kang P, Ding C, Zheng T, Wang X, Xiao Y. Excessive lodine Promotes Pyroptosis of Thyroid Follicular Epithelial Cells in Hashimoto's Thyroiditis Through the ROS-NF-KB-NLRP3 Pathway. Front Endocrinol (Lausanne) 2019; 10:778-778.

24. Li W, He W, Xia P, Sun W, Shi M, Zhou Y, Zhu W, Zhang L, Liu B, Zhu $J$ et al. Total Extracts of Abelmoschus manihot L. Attenuates Adriamycin-Induced Renal Tubule Injury via Suppression of ROS-ERK1/2-Mediated NLRP3 Inflammasome Activation. Front Pharmacol 2019; 10: 567-567.

25. Liu X, Zhang Z, Ruan J, Pan Y, Magupalli VG, Wu H, Lieberman J. Inflammasome-activated gasdermin $D$ causes pyroptosis by forming membrane pores. Nature 2016; 535(7610): 153-158.

Trop J Pharm Res, May 2020; 19(5): 1036 